\title{
Multiple access protocol for supporting multimedia services in wireless ATM networks
}

\author{
Liu, Hong; Dittmann, Lars; Gliese, Ulrik Bo; Danielsen, Per Lander
}

Published in:

Conference Proceedings of VIC'99

Link to article, DOI:

10.1109/VETEC.1999.780527

Publication date:

1999

Document Version

Publisher's PDF, also known as Version of record

Link back to DTU Orbit

Citation (APA):

Liu, H., Dittmann, L., Gliese, U. B., \& Danielsen, P. L. (1999). Multiple access protocol for supporting multimedia services in wireless ATM networks. In Conference Proceedings of VIC'99 IEEE.

https://doi.org/10.1109/VETEC.1999.780527

\section{General rights}

Copyright and moral rights for the publications made accessible in the public portal are retained by the authors and/or other copyright owners and it is a condition of accessing publications that users recognise and abide by the legal requirements associated with these rights.

- Users may download and print one copy of any publication from the public portal for the purpose of private study or research.

- You may not further distribute the material or use it for any profit-making activity or commercial gain

- You may freely distribute the URL identifying the publication in the public portal 


\title{
MULTIPLE ACCESS PROTOCOL FOR SUPPORTING MULTIMEDIA SERVICES IN WIRELESS ATM NETWORKS
}

\author{
Hong Liu, Lars Dittmann, Ulrik Gliese, Per Danielsen \\ Research Center COM, Technical University of Denmark \\ Building 349, DK-2800 Lyngby, DENMARK \\ PH: +45 45253876 FAX: +45 45936581 \\ e-mail: hl@com.dtu.dk, ld@ com.dtu.dk,ug@com.dtu.dk,pd@com.dtu.dk
}

\begin{abstract}
The future broadband wireless asynchronous transfer mode (ATM) networks must provide seamless extension of multimedia services from the wireline ATM networks. This requires an efficient wireless access protocol to fulfill varying Quality-of-Service (QoS) requirements for multimedia applications. In this paper, we propose a multiple access protocol using centralized and distributed channel access control techniques to provide QoS guarantees for multimedia services by taking advantage of the characteristics of different kinds of ATM traffics. Multimedia traffic, including constant bit rate (CBR), variable bit rate (VBR) and available bit rate (ABR) with different kinds of applications, such as voice, video-conferencing, MPEG video and Web-browsing, is considered to evaluate the proposed protocol. Simulation results show that the proposed multiple access protocol can support a wide range of telecommunication applications at the same time with high efficiency and guaranteed QoS.
\end{abstract}

\section{INTRODUCTION}

Support of a wide range of multimedia services in the wireless communication networks is becoming increasingly important in modern society. One solution is to extend multimedia services from ATM based wireline B-ISDN to the broadband wireless ATM (WATM) (1), (2). Since ATM was designed for supporting real time VBR video together with $\mathrm{CBR}$ voice and $A B R$ data in a sufficient bandwidth environment, the major challenge for the extension of multimedia services to the wireless networks is how to meet the end-to-end QoS requirements and still obtain an efficient use of the limited bandwidth. This requires a good multiple access protocol.

Some multiple access schemes for WATM with guaranteed QoS have been published (3)-(6). It seems very difficult to achieve a high throughput while providing $\mathrm{Q} O S$ guarantees for $\mathrm{CBR}, \mathrm{VBR}$ and $\mathrm{ABR}$ connections at the same time.

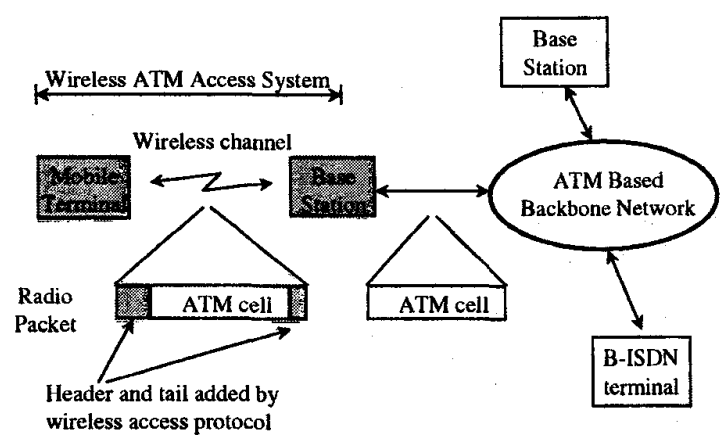

Fig. 1. Wireless extension of ATM network.

In this paper, a multiple access protocol is proposed on the basis of the broadband wireless ATM access system shown in Fig. 1. The proposed protocol uses centralized and distributed channel access control techniques to satisfy delay and jitter constraints for CBR and real time VBR (VBRrt) traffic, delay constrains for non real time VBR (VBRnrt) traffic and minimum cell rate demands for ABR traffic.

In the next section, the proposed multiple access protocol is described. In section III, simulation results for serving multimedia ATM traffics are presented to confirm the efficiency of the proposed protocol. Finally, the conclusions are drawn in section IV.

\section{MULTIPLE ACCESS PROTOCOL}

Multiple access protocol is designed to enable many users to share a common wireless channel with limited bandwidth. Those users are supposed to generate different kinds of traffics with different QoS demands. Therefore, a successful multiple access scheme should be able to take advantage of the characteristics of different ATM traffic to achieve high statistical multiplexing efficiency. Until now, three main techniques are used in the suggested multiple access 
protocols. These are polling (3), (4), contention and reservation (5)-(8). Reservation schemes work well for CBR traffic, while VBRrt traffic always brings every technique into trouble because of its burst and delay sensitivity.

The proposed access protocol is based on the packet reservation multiple access (PRMA) method (9), and the wireless access channel is a time division multiplexed (TDM) channel, where a frame structure with $\mathbf{N}$ identical time slots is used. Each time slot is used to transfer one packet containing one ATM cell and the media access control (MAC) protocol overhead.

Each time slot in the uplink channel is either reserved to a specific connection or available for contention. Slot reservations are controlled by the base station (BS), and the status of each uplink slot is announced through the downlink traffic. During the connection setup period, the mobile users provide information to the BS regarding the classes of traffic and their QoS parameters.

For CBR traffic, the mobile terminal is required to send the buffer delay of the current transmitting packet inband. Based on this, the BS will periodically assign one available time slot to the CBR terminal based on its peak cell rate, and adjust the assigned time slots interval according to the buffer delay and access delay of the current transmitted packet. The access delay is the elapsed time from when the slot is assigned till it is actually used for transmission. The time slot will be released after the CBR packet has been transmitted.

For VBR traffic, the mobile terminal (MT) dominates the channel access control. The MT sends an information packet for contention of an available time slot according to the number of packets stored in its buffer, its reserved slot number per frame and a permission parameter $p$. The permission parameter $p$ is related to the number of active VBR connections (10). Reservation of the time slot will be granted to the MT by the BS, if the contending packet is received without collision and transmission error. The maintaining and releasing of reserved slots in subsequent frames are also based on the number of packets stored in its buffer and its reserved slot number.

The restrictive reservation condition allows the VBR terminal to increase its bandwidth within a reasonable range according to its current cell rate and burst length. At the same time, the permission parameter $p$ reduces the probability of collision due to the contention. Thus, the distributed channel access technique can dynamically allocate the bandwidth to each VBR connection according to their current bursts and the traffic variation in the wireless channel, so that an efficient channel utilization is achieved.
For ABR traffic, the available bandwidth is predicted at the base station according to the reservation and collision in each frame. The base station will assign the available slots to each $A B R$ connection one after the other. The connection close to its minimum cell rate requirement will be served first, otherwise round-robin scheduling will be used for fairness.

\section{PERFORMANCE}

The broadband wireless ATM access system previously implemented is for a $32 \mathrm{Mbps}$ wireless channel supporting 25Mbps ATM traffic (10). Since the downlink is a broadcasting channel from a BS, only the uplink is considered in the simulations, where the traffic originates from various users with different traffic characteristics and QoS requirements. The frame structure used in the simulations is the same as that proposed in (10). The number of time slots per frame is 20 . The slot duration is about $17 \mu \mathrm{s}$.

Packet drop rate (PDR), mean transfer delay and channel utilization are used to evaluate the performance of the wireless access protocol. When the packet delay or jitter exceeds the maximum limits, it will be discarded. This dominates the packet drop rate. Especially, when very short maximum delay and jitter tolerances are used as QoS parameters, the packet drop rate due to the buffer length becomes insignificant.

Simulations are performed by combining CBR, VBR and $A B R$ traffic sources in two different scenarios. A CBR source is modeled by generating an ATM cell periodically according to its peak cell rate (PCR). A VBR source is characterized by PCR, sustainable cell rate (SCR) and maximum burst size (MBS). The model used for generating VBR traffic is the leaky-bucket. $A B R$ traffic is characterized by minimum cell rate (MCR). QoS parameters for CBR and VBR traffics include maximum cell transfer delay (MCTD) and maximum cell delay variation (MCDV).

\section{CBR, VBRrt and ABR Applications}

In this scenario, 10 video-conferencing connections, 16 Web-browsing connections and a variable number of MPEG video connections are served in the wireless channel. The video-conferencing connections require CBR with $\mathrm{PCR}=256 \mathrm{kbps}$, $\mathrm{MCTD}=5 \mathrm{~ms}$ and MCDV $=0.36 \mathrm{~ms}$. The Web-browsing connections need ABR service with $M C R=56 \mathrm{kbps}$. The MPEG video connections request VBRrt with $\mathrm{SCR}=1.5 \mathrm{Mbps}$, $\mathrm{PCR}=10 \mathrm{Mbps}, \quad \mathrm{MBS}=8 \mathrm{cells}, \quad \mathrm{MCTD}=5 \mathrm{~ms}$ and $\mathrm{MCDV}=1.5 \mathrm{~ms}$ 


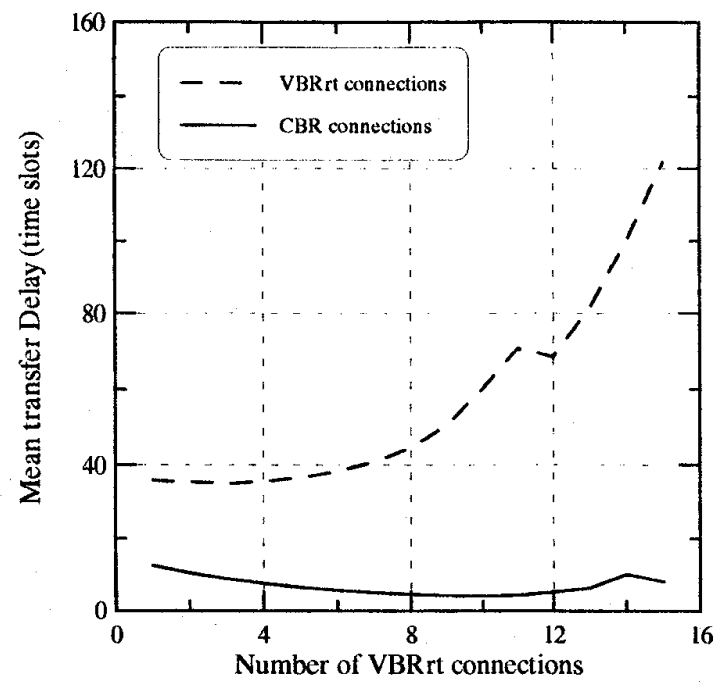

Fig. 2. Mean delay for CBR and VBRrt connections.

Figure 2 shows the mean transfer delay of CBR and VBRrt connections versus the number of MPEG video connections. Because the proposed multiple access protocol operates differently for CBR and VBR connections, and sets the highest priority to CBR connections, the mean delay of CBR connections is extremely low, and is getting lower when more VBR connections are connected. This is due to the increased collision from VBRrt connections so that more time slots are available for reservation. When the wireless channel is getting heavily loaded, the VBRrt connections will suffer from high transfer delay.

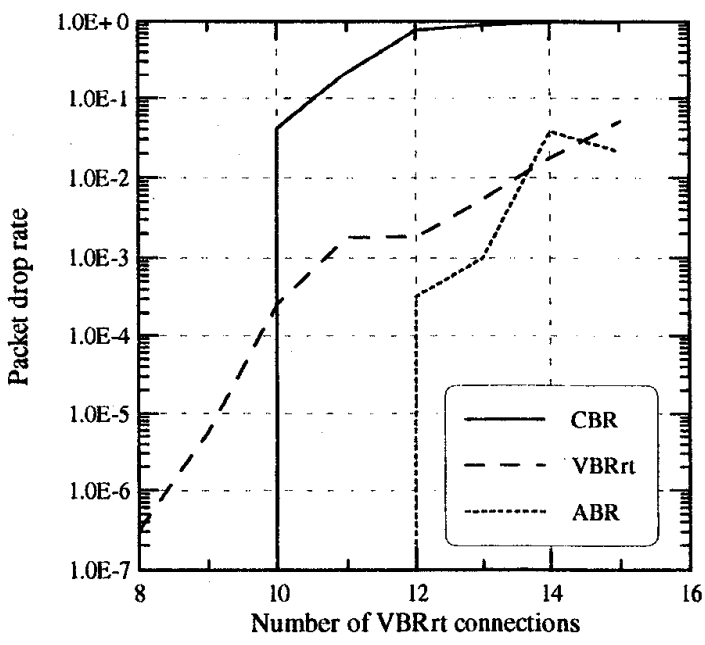

Fig. 3. PDR for CBR, VBRrt and ABR connections.

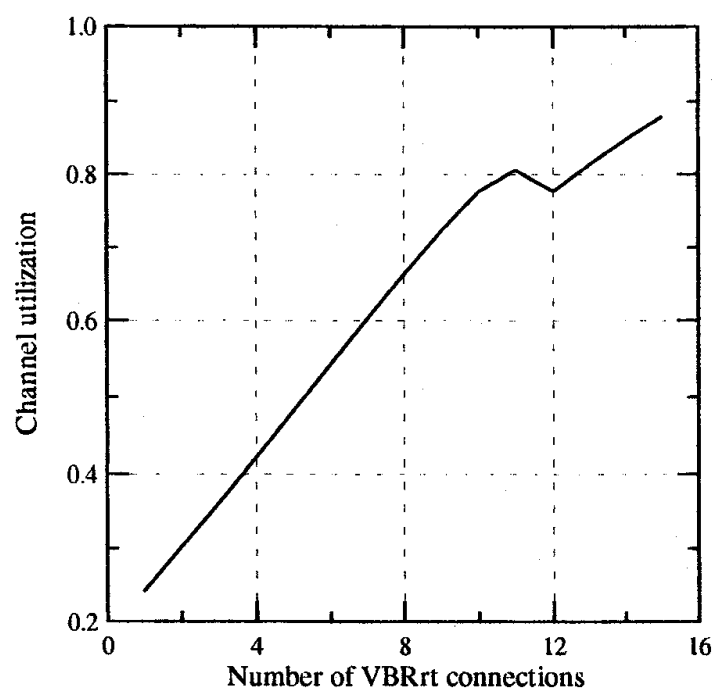

Fig. 4. Channel utilization versus the channel traffic load.

The packet drop rate for CBR and VBR connections against the number of VBRrt connections is given in Fig. 3. If the $P D R$ requirements of $C B R$ videoconferencing is $10^{-8}$, VBRrt MPEG video is $10^{-5}$, and ABR Web-browsing is $10^{-8}$, it can be seen that the proposed protocol may support ten $256 \mathrm{kbps}$ CBR connections, sixteen $56 \mathrm{kbps}$ ABR connections in combination with nine $1.5 \mathrm{Mbps}$ VBRrt connections with guaranteed $\mathrm{QoS}$ at the same time. In this case, the wireless channel utilization achieves more than $70 \%$, which is shown in Fig.4.

The reason of such high channel utilization is that the protocol works properly by taking advantage of the characteristic of different ATM traffics. It also sets constraints for VBR connections contending for an available time slot and keeping reservation, which results in lower collision probability, longer time for keeping a reserved time slot, and more radio resources available for the users who need them. But, Fig. 3 also illustrates that the VBRrt traffic plays an important role in the system performance because of its high traffic load, unexpected burst and stringent delay and jitter constraints.

\section{CBR, VBRnrt and ABR Applications}

Under this scenario, voice, video-on-demand and Webbrowsing services are used to evaluate the multiple access protocol. Voice connections generate CBR traffic with $\mathrm{PCR}=64 \mathrm{kbps}, \mathrm{MCTD}=5 \mathrm{~ms}$ and $\mathrm{MCDV}=0.36 \mathrm{~ms}$, while those video connections request VBRnrt with $\mathrm{PCR}=10 \mathrm{Mbps}, \quad \mathrm{SCR}=1.5 \mathrm{Mbps}$, $\mathrm{MBS}=8 \mathrm{cells}, \mathrm{MCTD}=30 \mathrm{~ms}$ and $\mathrm{MCDV}=2.3 \mathrm{~ms}$. Web- 


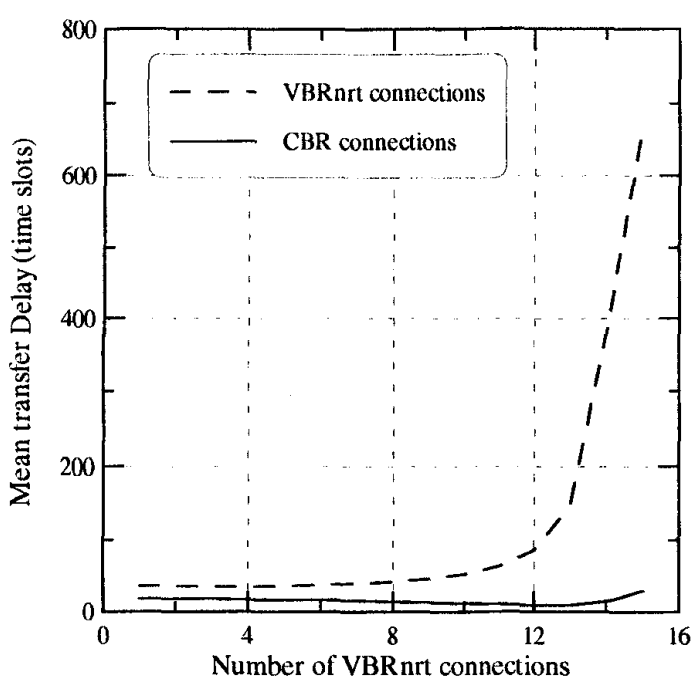

Fig. 5. Mean delay for CBR and VBRnrt connections.

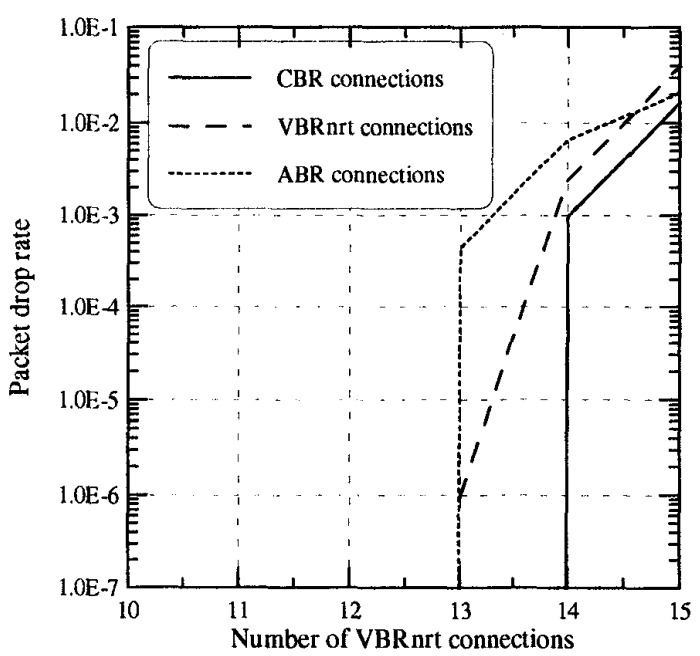

Fig. 6. PDR for CBR, VBRnrt and ABR connections.

browsing service is the same as mentioned in the above scenario. During the simulation period, 30 voice connections and 10 Internet connections are connected. Video-on-demand connection is added to the wireless network one by one. The main difference between the two scenarios is that the VBR traffic demands loose delay and jitter constraints in this scenario.

Figure 5 shows the mean transfer delay versus the number of 1.5Mbps VBRnrt connections. It is observed that the mean delay of CBR connections still keeps very low, while in the heavy load situation, the mean delay of VBRnrt connections is higher than that of VBRrt

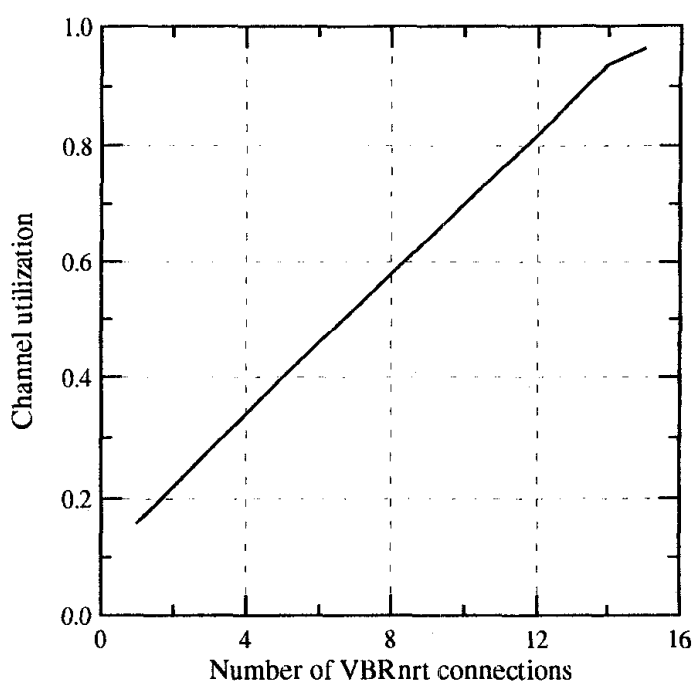

Fig. 7. Channel utilization versus the channel traffic load.

connections in the first scenario, because VBRnrt can tolerate higher delay.

Since ABR traffic get the lowest priority to access the wireless channel, when VBR traffic can tolerate higher delay, ABR connections performance will dominate the whole performance. This is illustrated in Fig. 6. This phenomenon may also mean that ABR traffic can be treated as VBRnrt traffic in the proposed protocol, which will not degrade the performance of the proposed access protocol.

Figure 6 together with Fig. 7 shows that if the delay and jitter constraints of VBR traffic can be loosed, the channel utilization can go up to more than $80 \%$ while maintaining the QoS requirements of all the ATM traffics.

\section{CONCLUSIONS}

In this paper, a multiple access protocol was proposed for providing guaranteed QoS for all kinds of ATM traffics in the wireless ATM networks. This protocol takes full advantage of the traffic characteristics of different kinds of ATM traffics to achieve high statistical multiplexing efficiency, using central controlled resources assignment scheme for CBR traffic and distributed reservation and contention techniques for VBR traffic, and giving unused radio resources to ABR connections with fairness.

The protocol tries to meet the very stringent delay and delay variation requirements of CBR and VBR traffics, and the low packet drop rate of ABR traffic. Two kinds 
of combinations of voice, video-conferencing, MPEG video, video-on-demand and Web-browsing applications are investigated in the simulations to evaluate the system performance. Simulation results show that the proposed protocol can be used for serving a wider range of telecommunication applications with guaranteed QoS in the wireless ATM networks, and achieve high wireless channel utilization.

\section{REFERENCES}

(1) Ayanoglu, E., Eng, K.Y., and Karol, M.J., "Wireless ATM: limits, challenges and proposals," IEEE Personal Communications, Vol. 3, No. 4, 1996, pp. 18-34.

(2) Raychaudhuri, D., and Wilson, N.D., "ATM-based transport architecture for multiservices wireless personal communication networks," IEEE JSAC, Vol. 12, No. 8, October 1994, pp. 1401-1414.

(3) Chang, C., Chen, K.-C., You, M., and Chang, J., "Guaranteed Quality-of-Service Wireless Access to ATM Networks," IEEE JSAC, Vol. 15, No. 1, January 1997, pp. 106-118.

(4) Passas, N., Paskalis, S., Vali, D., and Merakos, L., "Quality-of-Service-Oriented medium access control for wireless ATM networks," IEEE Communications Magazine, November 1997, pp. 42-50.
(5) Biswas, S.K., Reininger, D., and Raychaudhuri, D., "UPC Based Bandwidth Allocation for VBR Video in Wireless ATM Links," Proc. ICC'97, May 1997, pp. 1073-1079.

(6) Naghshineh, M., and Acampora, A.S., "QOS provisioning in micro-cellular networks supporting multimedia traffic," IEEE Infocom 95, April 1995.

(7) Kim, J.G., and Widjaja, I., "PRMADDA: A new media access control protocol for wireless ATM," Proc. ICC'96, may 1996, pp. 240-244.

(8) Nørskov, S., Gliese, U. and Stubkjær, K.,"Adaptive packet reservation multiple access (A-PRMA) for broadband wireless ATM," Selected papers on mobile multimedia communications, Plenum Publishing Corp., NY, 1997, ISBN 0-306-457725, pp. 167-171.

(9) Nanda, S., Goodman, D.J., and Timor, U., "Performance of PRMA: A Packet Voice Protocol for Cellular Systems," IEEE Trans. on Vehicular Tech., Vol. 40, No. 3, Aug 1991, pp.584-598.

(10)Le, K.H., Nørskov, S., Dittmann, L., and Gliese, U., "Base Station MAC with APRMA Protocol for Broadband Multimedia ATM in micro/pico Cellular Mobile Networks," Proc. VTC'98, may 1998, pp234238. 